\title{
Development of a blended learning curriculum to improve POCUS education in a pediatric emergency medicine training program
}

\author{
Maya Harel-Sterling ${ }^{1} \mathbb{D} \cdot$ Lianne J McLean $^{1}$
}

Received: 1 September 2021 / Accepted: 7 January 2022 / Published online: 25 January 2022

(c) The Author(s), under exclusive licence to Canadian Association of Emergency Physicians (CAEP)/ Association Canadienne de Médecine d'Urgence (ACMU) 2022

\begin{abstract}
We engaged in curriculum enhancement of POCUS training in our pediatric emergency medicine subspecialty training program using Kern's model of curricular development. We enhanced existing training to include focused asynchronous learning of image interpretation, longitudinal scanning shifts, and quarterly progress report score cards and check-ins for improved communication, transparency and reinforcement of goals. Our approach could inform other training programs looking to improve their POCUS curricula.
\end{abstract}

Keywords Point-of-care ultrasound $\cdot$ Medical education $\cdot$ Pediatric emergency medicine

\section{Résumé}

Nous travaillons sur l'amélioration du curriculum de la formation d'échographie d'urgence de notre programme d'urgences pédiatriques en utilisant le modèle de développement de curriculum de Kern. Nous avons amélioré la formation existante pour y inclure un apprentissage asynchrone ciblé de l'interprétation des images, des sessions pratiques d'échographie longitudinales, ainsi que des cartes de pointage et des contrôles trimestriels pour améliorer la communication, la transparence et le renforcement des objectifs. Notre approche pourrait être utile à d'autres programmes de formation cherchant à améliorer leurs programmes d'échographie d'urgence.

\section{Background}

Point-of-care ultrasound (POCUS) is commonly used at the bedside in the pediatric emergency department (PED), and has been shown to expedite patient care and improve health outcomes [1]. There are increasing expectations in general emergency medicine and pediatric emergency medicine (PEM) programs that trainees obtain POCUS skills during their training [2]. A 2015 statement from the American Academy of Pediatrics highlighted the importance of POCUS education during residency training, and stated that PEM subspecialty residency programs should have a structured POCUS education curriculum and competency assessment for residents in-training [1]. However, the process of

Maya Harel-Sterling

maya.harel-sterling@ sickkids.ca

1 Division of Paediatric Emergency Medicine, Department of Paediatrics, The Hospital for Sick Children, Toronto, ON, Canada implementing these recommendations has been quite variable, and many residents struggle to achieve competency during their training [3, 4]. Previous studies have identified time allocation, lack of qualified faculty, and inadequate training in clinical integration as major barriers to achieving competency $[3,5]$. Despite having a 1-month focused experience in POCUS during training, in the preceding 2 years prior to our educational innovation, only 1 PEM resident had achieved credentialing in any core POCUS application.

\section{Purpose}

We undertook a curriculum enhancement project to improve POCUS training in our program using Kern's model of curriculum development. 


\section{Methods}

Beginning in July 2020, we restructured our POCUS curriculum using Kern's 6-step approach to curricular development [6]. We began with problem identification, followed by a needs assessment, development of goals and objectives, selecting educational strategies, implementation and evaluation [6].

\section{Results}

\section{Problem identification}

Prior to our educational innovation, we had a pre-existing POCUS program for PEM residents including a 1-month mandatory rotation in POCUS and Diagnostic Imaging, and optional online resources. However, there was a lack of a formalized curriculum, and these resources were not universally accessed.

\section{Needs assessment}

A baseline needs assessment of 13 PEM residents who had completed their training between July 2017 and June 2020 was performed. With regards to the importance of learning POCUS for their everyday practice, $29.2 \%$ rated the skill as "extremely important", 33.3\% as "very important", and $27 \%$ as "somewhat important". While they were motivated to learn this skill, they reported multiple barriers to getting credentialed during their PEM residency training, including lack of a formalized curriculum, time pressures and high workload, and not always having a POCUS-trained attending available to review with on shift.

\section{Goals and objectives}

Given the limited Royal College objectives specific to POCUS in Pediatric emergency medicine training [7], we pulled from previous consensus statements about the core POCUS applications that should be taught in PEM training [8]. Core applications for our program include lung, cardiac, e-FAST, soft tissue. For each POCUS application, the task is broken down into smaller components: image acquisition, interpretation, understanding indications and clinical integration.

\section{Educational strategies and implementation}

We enhanced the existing POCUS training curriculum for PEM subspecialty residents using a blended learning approach (Fig. 1).

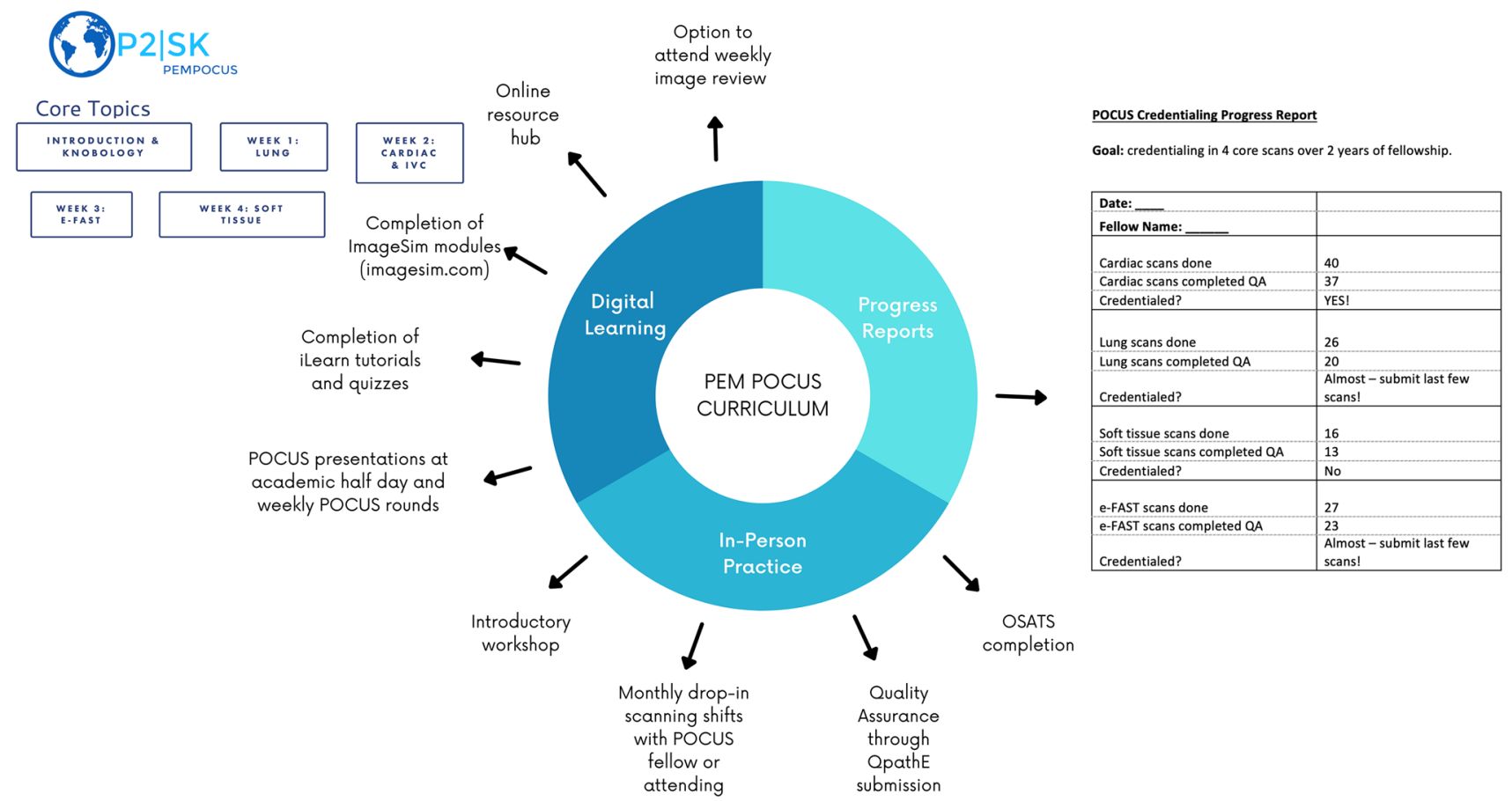

Fig. 1 A blended learning PEM POCUS curriculum, including digital learning, in-person practice and progress reports 


\section{Description of the innovation}

\section{Online resources}

We organized the available asynchronous online resources through a program website (p2sk.ca) to encourage their use. These resources focus on image interpretation and clinical integration. Synchronous online learning opportunities were also made available to residents including weekly image review, weekly POCUS rounds, and regular presentations at academic half-day.

\section{Longitudinal scanning shifts}

Monthly drop-in scanning shifts were offered with a POCUS fellow or attending. Given that individual learners have their own learning curves [9], this allowed the skill to be learned at their own pace, and to reinforce learning using spaced repetition [10]. Scans are archived on a cloud-based system (QpathE) and are regularly reviewed for quality assurance (QA) by a POCUS fellow or attending for additional feedback.

\section{POCUS progress reports}

We created POCUS progress report score cards for each resident (Fig. 1) that were updated on a quarterly basis. These highlighted the number of scans performed and number with completed QA for each of the 4 core applications. The PEM residents reviewed these with their program director during their scheduled residency feedback meetings to reinforce and encourage their progress, and to help foster a conversation about their individual goals and objectives.

\section{Program evaluation}

Following Kirkpatrick's hierarchy [11], our program evaluation occurred at multiple levels. Knowledge transfer and image interpretation were tested using the iLearn quizzes (passing score of 90\%), and ImageSim. Image acquisition was evaluated using an Objective Structured Assessment of Technical Skills (OSATS) during scanning shifts. Changes in behavior were evaluated by monitoring the monthly number of scans performed and submitted for QA by each resident. Results were assessed by the number of PEM residents becoming credentialing in the core POCUS applications. To become credentialed, they must complete and pass the iLearn quizzes, submit 25 scans per application for QA, and complete an OSATS $[8,12,13]$.

In the 1 year since our educational innovation, the mean number of POCUS scans performed per resident increased from a mean $(\mathrm{SD})$ of $3.5( \pm 1.4)$ scans/month (July-September 2020) to 10.9 ( \pm 1.4$)$ scans/month (April-June 2021). The number of fellows scanning per month ranged from 5 to 9 . On average, $81 \%$ of the PEM residents' scans were submitted for QA. Overall, 5/10 PEM residents have become credentialed in at least one core application (range of 2-4 applications each). All 5 became credentialed in cardiac and e-FAST, 3 in lung, and 2 in soft tissue, for a total for 15 credentialed applications among them.

\section{Discussion}

Through curriculum enhancement of our POCUS training using Kern's model, we were able to dramatically increase the number of scans performed by trainees and the number of trainees achieving credentialing. By implementing longitudinal scanning opportunities, regular POCUS presentations at academic half-day, quizzes, and quarterly progress reports we utilized spaced repetition [10], interleaving [10] and self-regulated learning theories [14], respectively. Other PEM or EM training programs could use a similar approach to inform their own curriculum enhancement. Future program evaluation will include both quantitative assessments with tracking of PEM resident scanning and credentialing over time, as well as qualitative assessments of resident satisfaction and confidence in their POCUS skills, through surveys and focus groups.

\section{Limitations}

Our program was facilitated by an established POCUS fellowship affiliated with our PED. Smaller programs and institutions may be limited by lack of POCUS expertise and access to ultrasound machines and infrastructure. However, the shift to online asynchronous resources can assist with this knowledge transfer to smaller institutions.

\section{Summary}

By using an established framework in curriculum development, we were able to improve POCUS education in a PEM training program.

Author contributions All of the authors are responsible for the described educational innovation project. They have all contributed to the concept and design, description of the innovation and reporting of the data, drafting or revising of the manuscript, and have approved the manuscript as submitted. 
Funding Not applicable.

Availability of data and materials (data transparency) Not applicable.

Code availability (software application or custom code) Not applicable.

\section{Declarations}

Conflict of interest The authors have no conflicts of interest to declare.

Ethical approval This work was approved as part of a Quality Improvement project through the Hospital for Sick Children Quality Management Department.

\section{References}

1. Marin JR, Lewiss RE. Point-of-care ultrasonography by pediatric emergency medicine physicians. Pediatrics. 2015;135(4):e1113-22.

2. Lewis D, Rang L, Kim D, Robichaud L, Kwan C, Pham C, et al. Recommendations for the use of point-of-care ultrasound (POCUS) by emergency physicians in Canada. CJEM. 2019;21(6):721-6.

3. Acuña J, Rubin M, Hahn B, Das D, Kapoor M, Adhikari S, et al. Point-of-care ultrasound in United States pediatric emergency medicine fellowship programs: the current state of practice and training. Pediatr Emerg Care. 2020. https://doi.org/10.1097/PEC. 0000000000001955.

4. Hoeffe J, Desjardins MP, Fischer J, Carriere B, Gravel J. Emergency point-of-care ultrasound in Canadian pediatric emergency fellowship programs: current integration and future directions. Can J Emerg Med. 2016;18:469-74.
5. Haney RM, Halperin M, Diamond E, Ratanski D, Shokoohi H, Huang $\mathrm{C}$, et al. Clinical integration of point-of-care ultrasound by emergency medicine residents: a single-center mixed-methods study. AEM Educ Train. 2020;4(3):212-22.

6. Thomas PAKD, Hughes MT, Chen BY. Curriculum development for medical education: a six-step approach. Baltimore: Johns Hopkins University Press; 2015. p. 300.

7. Royal College of Physicians and Surgeons of Canada. Objectives of Training in the Subspecialty of Pediatric Emergency Medicine. 2013. Available at: https://www.royalcollege.ca/rcsite/documents/ $\mathrm{ibd} /$ pediatric-emergency-medicine-otr-e.pdf. Accessed $28 \mathrm{Oct}$ 2021.

8. Shefrin AE, Warkentine F, Constantine E, Toney A, Uya A, Doniger SJ, et al. Consensus core point-of-care ultrasound applications for pediatric emergency medicine training. AEM Educ Train. 2019;3(3):251-8.

9. Kwan C, Pusic M, Pecaric M, Weerdenburg K, Tessaro MA-O, Boutis KA-O. The variable journey in learning to interpret pediatric point-of-care ultrasound images: a multicenter prospective cohort study. AEM Educ Train. 2020;4:111-22.

10. Brown PC, Roediger HL, McDaniel MA. Make it stick: the science of successful learning. Cambridge: Harvard University Press; 2014.

11. Kirkpatrick D, Kirkpatrick J. Evaluating training programs: the four levels. Oakland: Berrett-Koehler Publishers, Incorporated; 2006.

12. Abo AM, Alade KH, Rempell RG, Kessler D, Fischer JW, Lewiss $\mathrm{RE}$, et al. Credentialing pediatric emergency medicine faculty in point-of-care ultrasound: expert guidelines. Pediatr Emerg Care. 2019. https://doi.org/10.1097/PEC.0000000000001677.

13. Guidelines U. Emergency, point-of-care and clinical ultrasound guidelines in medicine. Ann Emerg Med. 2017;69(5):e27-54.

14 White CB, Gruppen LD, Fantone JC. Self-regulated learning in medical education. Chichester: Wiley; 2013. p. 201-11. 